\title{
Flame spread along a fuel rod in the absence of gravity
}

\author{
F J Higuera and A Liñán
}

\begin{abstract}
An analysis is carried out of the steady spread of a flame with infinitely fast kinetics over a thermally thin solid rod in the absence of gravity. Due to the radial convergence on the solid surface of the heat flux heating and vaporizing the fuel, the spread rate is found to be higher than for a flame spreading over a slab, and the region of the gas around the flame which is affected by molecular transport is large compared with the radius of the rod. The effects of radiation, of a buoyancy force opposing flame propagation and of axial heat conduction in the solid are discussed briefly.
\end{abstract}

\section{Introduction and orders of magnitude}

Cylindrical solid fuel samples have been used in experimental and theoretical studies of steady combustion and flame propagation (see, e.g., [1-8]) both because they present practical experimental advantages over planar samples and because the real elements whose burning characteristics some of these experiments are aimed at elucidating are actually cylindrical. In particular, this is the case for electric cables, which are deemed potentially dangerous elements from the point of view of fire safety owing to the combination of a combustible plastic coating and a highly conducting metallic core. Cylindrical samples are also used in experiments on flame spread in microgravity [9], where their practical advantages are made more prominent by the space limitations typically imposed on such experimental configurations. In many of these experiments, when no flow aiding or opposing flame propagation exists, the size of the flame front is observed to be large compared with the radius of the sample. The consequences of this scale disparity, which is specific to microgravity environments, will be analysed in the present work.

The spread of a gas-phase flame over a solid fuel requires that part of the heat released by the flame be transferred to the cold solid ahead of the flame to supply the energy necessary for heating and vaporizing the fuel. The rate of heat transfer, and therefore the flame spread rate, depends on the motion of the gas relative to the advancing flame. In the absence of gravity and forced convection, the flow velocity seen by the flame front is of the order of its propagation speed along the solid, $V_{p}$, and molecular transport in the gas extends to a region of characteristic size $l_{g}=\alpha_{g} / V_{p}$ around the flame front, where $\alpha_{g}=k_{g} / \rho_{g} c_{p}$ is the thermal diffusivity of the gas and $k_{g}, \rho_{g}$ and $c_{p}$ are its conductivity, density and specific heat, respectively. In the much studied case of a two-dimensional flame spread over a slab, the conduction heat flux reaching the solid surface in the region of size $l_{g}$ is of the order of $q_{g}=k_{g} \Delta T_{g} / l_{g}$, where $\Delta T_{g}$ is the characteristic gas-phase temperature variation, equal to the difference between the adiabatic flame temperature $T_{f}$ and the ambient temperature $T_{\infty}$. Advancing that the slab will behave as thermally thin for steady flame spread in the absence of 
gravity (i.e. that $a \ll\left(\alpha_{s} l_{g} / V_{p}\right)^{1 / 2}$, where $a$ and $\alpha_{s}=k_{s} / \rho_{s} c_{s}$ are the thickness and thermal diffusivity of the solid and $k_{s}, \rho_{s}$ and $c_{s}$ denote its conductivity, density and specific heat, respectively), so that the temperature is transversally uniform, an energy balance requires that the total heat received by the non-vaporizing surface, of the order of $q_{g} l_{g}=k_{g} \Delta T_{g}$ in the absence of radiation, be equal to $\rho_{s} c_{s} a V_{p} \Delta T_{s}$, where $\Delta T_{s}$ is the difference between the solid vaporization temperature $T_{v}$ and the ambient temperature. This balance yields the order of magnitude estimate $V_{p}=\varepsilon^{2} \alpha_{g} / a$, with $\varepsilon=\left(\rho_{g} c_{p} \Delta T_{g} / \rho_{s} c_{s} \Delta T_{s}\right)^{1 / 2}$, which coincides with the classic formula of de Ris [10] up to a numerical factor. The parameter $\varepsilon$ is typically small compared with unity, due to the small value of the density ratio. Using these results, $l_{g}=a / \varepsilon^{2} \gg a$ and $a /\left(\alpha_{s} l_{g} / V_{p}\right)^{1 / 2}=\mathrm{O}\left(\varepsilon N^{1 / 2}\right)$, where $N=\left(k_{g} \Delta T_{g} / k_{s} \Delta T_{s}\right)$ and the product $\varepsilon N^{1 / 2}$ is also typically small, which justifies the assumption of a thermally thin solid.

Bhattacharjee and Altenkirch [11,12] have shown that surface and gas radiation are important in microgravity conditions, leading to a class of flames that are radiatively controlled and can extinguish at low velocities, in agreement with experimental observations [13]. Unsteady flame spread and extinction over thermally thick solids in reduced gravity has been studied in [14]. The ratio of surface radiation to gas-to-surface conduction is measured by $R_{s}=\sigma e_{s}\left(T_{v}^{4}-T_{\infty}^{4}\right) l_{g} /\left(k_{g} \Delta T_{g}\right)$, where $e_{s}$ and $\sigma$ are the surface emissivity and the StefanBoltzmann constant. This ratio increases with $l_{g}$, leading to a reduction of the net energy flux reaching the surface and thus of the spread rate $V_{p}$, which in turn increases $l_{g}$; see $[11,12]$. The energy radiated by the hot gas in a region of size $l_{g}$ around the flame front is of the order of $\sigma a_{g}\left(T_{f}^{4}-T_{\infty}^{4}\right) l_{g}^{2}$ per unit length along the front, where $a_{g}$ is an absorption coefficient and the gas is assumed to be optically thin. An order unity fraction of this energy is absorbed by the solid, enhancing flame spread when $R_{g}=\sigma a_{g}\left(T_{f}^{4}-T_{\infty}^{4}\right) l_{g}^{2} /\left(k_{g} \Delta T_{g}\right)=\mathrm{O}(1)$, while the rest is lost to the environment, along with the energy radiated by the surface, which decreases the flame temperature and the spread rate.

Some of the foregoing estimates require modification in the case of a flame propagating along a rod of radius $a$ in the absence of gravity and forced convection. Consider first the conduction heat flux reaching the solid. Advancing that, as before, $l_{g} \gg a$, the thin, cold rod is immersed in a gas at a high temperature, of the order of the flame temperature up to distances of $\mathrm{O}\left(l_{g}\right)$ from the surface. In these conditions radial convection and conduction dominate in the gas around the rod, leading to a heat flux of the order of $q_{g}=k_{g} \Delta T_{g} / a$ at the surface. The energy balance $q_{g} l_{g} a \sim \rho_{s} c_{s} a^{2} V_{p} \Delta T_{s}$ (for a thermally thin rod), with $l_{g}=\alpha_{g} / V_{p}$, now yields the modified estimate of the spread rate $V_{p}=\varepsilon \alpha_{g} / a$, which, while still inversely proportional to $a$, is larger than the estimate for a slab by a factor $\varepsilon^{-1}$. This is an important geometrical effect due to the radial convergence of the heat flux on the solid surface. The result also implies that $l_{g}=a / \varepsilon \gg a$, as was advanced before, and that $a /\left(\alpha_{s} l_{g} / V_{p}\right)^{1 / 2}=\mathrm{O}\left(N^{1 / 2}\right)$, so that the solid behaves as thermally thin if $N$ is small compared with unity. If, on the other hand, $N \gg 1$, then the solid behaves as thermally thick, conduction extending only to a thermal layer of thickness $\delta_{s} \sim\left(\alpha_{s} l_{g} / V_{p}\right)^{1 / 2}$, and the energy balance $k_{g} \Delta T_{a} / a \sim k_{s} \Delta T_{s} / \delta_{s}$, with $l_{g}=\alpha_{g} / V_{p}$, gives $l_{g}=a /\left(\varepsilon N^{1 / 2}\right)$ and $V_{p}=N^{1 / 2} \varepsilon \alpha_{g} / a$. All these estimates are accurate up to logarithms of $\varepsilon$.

The ratio of surface radiation to gas-to-surface conduction is now measured by $R_{s}^{\prime}=$ $\sigma e_{s}\left(T_{v}^{4}-T_{\infty}^{4}\right) a /\left(k_{g} \Delta T_{g}\right)$, smaller by a factor $\varepsilon^{-1}$ than the ratio $R_{s}$ for a slab and independent of gravity and forced flow, whose effects entered through $l_{g}$ in the latter case, insofar as $l_{g} \gg a$. On the other hand, only a fraction of order $\varepsilon$ of the energy radiated by the gas in a volume of order $l_{g}^{3}$ around the flame front reaches the surface, owing to geometrical effects, so that the ratio of gas-to-surface radiation to conduction is $R_{g}^{\prime}=\sigma a_{g}\left(T_{f}^{4}-T_{\infty}^{4}\right) l_{g} a /\left(k_{g} \Delta T_{g}\right)=\varepsilon R_{g}$. These results suggest that the effect of radiation on the energy balance at the surface is much smaller for a rod than for a slab. This effect will be neglected in what follows. Radiative 
losses from the gas to the environment, which are of the same order in the two cases, might be accounted for by means of an effective reduction of the heat released at the flame, while a more accurate treatment, which will not be undertaken here, could proceed along the lines of [12]. Extended estimates accounting for the effects of buoyancy, forced flow and finite rate kinetics are given in [9], but all of these effects will be left out in the remainder of this paper, restricting the analysis to the simplest possible problem featuring geometrical convergence.

\section{Formulation}

In the Burke--Schumann limit of infinitely fast chemical reaction, neglecting flame and solid radiation, and assuming that the vaporization of the solid occurs at a constant temperature and that the Lewis numbers of the gas-phase reactants are equal to unity, the flow around a flame front propagating steadily along a thermally thin rod is determined by the solution of the following nondimensional problem:

$$
\left.\begin{array}{l}
\nabla \cdot(\rho v)=0 \quad \rho v \cdot \nabla v=-\nabla p+\operatorname{Pr} \nabla \cdot \tau^{\prime} \\
\rho v \cdot \nabla(Z, H)=\nabla \cdot[\tilde{k} \nabla(Z, H)] \\
Z=\frac{s Y_{F}-Y_{O}+1}{1+s} \quad H=T-1+\gamma\left(Y_{F}+Y_{O}-1\right) \\
Y_{F} Y_{O}=0 \quad\{T=1
\end{array}\right\} \begin{aligned}
& u=U \quad \rho v=m \quad m Z-\tilde{k} \frac{\partial Z}{\partial r}=m \quad T=T_{s} \\
& m H-\tilde{k} \frac{\partial H}{\partial r}=-m \tilde{L}-\frac{\gamma-\tilde{L} /(1+s)}{2 \varepsilon^{2}\left(T_{v}-1\right)} U \frac{\mathrm{d} T_{s}}{\mathrm{~d} x} \\
& (-x, r) \rightarrow \infty: \quad v=Z=H=0 \quad T_{s}=1
\end{aligned}
$$

where the variables $\left(\boldsymbol{x}, \boldsymbol{v}, p, \rho, T, T_{s}, Y_{F}, Y_{O}, m\right)$ are nondimensionalized with the factors $\left(a, \alpha_{g} / a, \rho_{g} \alpha_{g}^{2} / a^{2}, \rho_{g}, T_{\infty}, T_{\infty}, 1, Y_{O \infty}, \rho_{g} \alpha_{g} / a\right)$, with $\rho_{g}, T_{\infty}, \alpha_{g}$ and $Y_{O \infty}$ denoting the ambient gas density, temperature, thermal diffusivity and oxygen mass fraction, respectively. Here $\boldsymbol{x}=(x, r)$ are cylindrical coordinates, $x$ being the distance along the axis of the rod measured from the vaporization inception section. $v=(u, v)$ are the corresponding velocity components and $m(x)$ is the vaporization flux, equal to zero for $x<0$, where the nondimensional temperature of the solid $T_{s}(x)$ is smaller than the nondimensional vaporization temperature $T_{v}$ (scaled with $T_{\infty}$ ), and greater than zero for $x>0$, where $T_{s}=T_{v}$. The variation of the radius of the vaporizing rod has been neglected in (1)-(5) for reasons that will be discussed below. In what follows $\tilde{k}=k_{g} / k_{g}\left(T_{\infty}\right)=T^{1 / 2}$. Problem (1)-(5) contains the six nondimensional parameters $s, \gamma=Q /\left[c_{p} T_{\infty}(1+s)\right], T_{v}, \tilde{L}=L /\left(c_{p} T_{\infty}\right)-T_{v}+1, P r$ and $\varepsilon=\left[\rho_{g} c_{p}\left(T_{f}-1\right) / \rho_{s} c_{s}\left(T_{v}-1\right)\right]^{1 / 2}$, where $s, Q$ and $L$ are the air-to-fuel mass stoichiometric ratio, the heat released by the flame per unit mass of fuel and the effective vaporization heat, respectively; $T_{f}=1+\gamma-\tilde{L} /(1+s)$ is the adiabatic flame temperature scaled with $T_{\infty}$ and $\mathrm{Pr}$ is the Prandtl number of the gas. The nondimensional propagation speed $U$, which we anticipate to be of $\mathrm{O}(\varepsilon)$ on the basis of the estimates of the previous section, is an eigenvalue to be determined as a function of these parameters with the condition that $T_{s}(x)$ be continuous at the vaporization front: $T_{s} \nearrow T_{v}$ when $x \nearrow 0$.

In the asymptotic limit $\varepsilon \rightarrow 0$, the solution of (1)-(5) consists of two regions: an outer region where $(x, r)=\mathrm{O}\left(\varepsilon^{-1}\right),(u, v)=\mathrm{O}(\varepsilon)$ and the whole conservation equations must be solved, and an inner region where $x=\mathrm{O}\left(\varepsilon^{-1}\right), r=\mathrm{O}(1)$, the flow is nearly radial $(v=\mathrm{O}(1) \gg(u, U)=\mathrm{O}(\varepsilon))$ and only radial convection and diffusion matter in the first 
approximation. At leading order in this latter region, $u=0, \rho v=m / r, Z=1-a(x) \mathrm{e}^{m \xi}$ and $H=-\tilde{L}+b(x) \mathrm{e}^{m \xi}$ for $x>0$, with $a \gamma(1+s) / s+b=\tilde{L}+T_{v}-1$, and $u=v=0$, $Z=d(x)$ and $H=\xi[\gamma-\tilde{L} /(1+s)] /\left[2 \varepsilon^{2}\left(T_{v}-1\right)\right] \mathrm{d} T_{s} / \mathrm{d} x+e(x)$ for $x<0$, with $(1-d) \gamma(1+s) / s+e=T_{s}-1$ if $d>1 /(1+s)$ and $\gamma(1+s) d+e=T_{s}-1$ otherwise. Here $\xi=\int_{1}^{r} \rho^{1 / 2} \mathrm{~d} r / r$ and the functions $a(x), d(x)$ and $m(x)$ must be determined by matching with the outer region. In particular, matching requires $m=\mathrm{O}(-1 / \ln \varepsilon)$. For $\varepsilon x \rightarrow \infty$, axial diffusion and heat conduction can be neglected, the mixture fraction $Z$ and the enthalpy $H$ become essentially functions of $U^{1 / 2} r / x^{1 / 2}$, and the vaporization flux takes on the asymptotic form

$$
m \sim T_{v}^{1 / 2} \frac{\ln \left([\tilde{L}+\gamma(1+s) / s] /\left(\tilde{L}+T_{v}-1\right)\right)}{\ln (x / U)^{1 / 2}} .
$$

The solution of (1)-(5) can be simplified by taking advantage of the fact that the ratio $\left(T_{v}-1\right) /\left(T_{f}-1\right)$ is often small. This means that the temperature variation in the solid, $\left(T_{s}-1\right)=\mathrm{O}\left(T_{v}-1\right)$, is small compared with the temperature variation in the gas, $(T-1)=\mathrm{O}\left(T_{f}-1\right)$, and therefore the condition $T=T_{s}$ at $r=1$ can be simplified to $T=1$ at $r=1$ when solving the gas problem. In addition, from the solid continuity equation $\left(\rho_{s} / \rho_{g}\right) U \mathrm{~d} a / \mathrm{d} x=-m$, the nondimensional burnout distance can be seen to be of the order of $\varepsilon^{-1}\left(T_{f}-1\right) /\left(T_{v}-1\right)$ if $\left(\tilde{L}+T_{v}-1\right) /\left(T_{f}-1\right)$ is not small. This distance is moderately large compared with the characteristic size $\varepsilon^{-1}$ of the flame front region, and therefore the radius of the rod and the density of the solid can be assumed to be constant when solving the problem in the flame front region, a simplification already included in the formulation (1)-(5). With these approximations, the problem (1)-(5) can be solved for given values of $s, \gamma, \operatorname{Pr}, \tilde{L}$ and $U$, using the boundary condition ( $4 b)$ only in the vaporizing region of the surface $x>0$, where $\mathrm{d} T_{s} / \mathrm{d} x=0$. The solution determines $\partial H / \partial r$ in the nonvaporizing region of the surface $x<0$, where $m=0$. Integrating then $(4 b)$ over this region yields

$$
\varepsilon=\left\{\frac{[\gamma-\tilde{L} /(1+s)] U}{2 I}\right\}^{1 / 2} \quad \text { with } \quad I=\left.\int_{-\infty}^{0} \tilde{k} \frac{\partial H}{\partial r}\right|_{r=1} \mathrm{~d} x .
$$

\section{Results and discussion}

The nondimensional spread rate obtained upon inversion of the relation $\varepsilon(U)$ from the numerical solution of the simplified form of (1)-(5) for $s=8, \tilde{L}=2$ and $\operatorname{Pr}=1$, corresponding approximately to the combustion of PMMA in air [15], is given in figure 1 as a function of $\varepsilon$ for three different values of $\gamma$. The relatively weak dependence of $U$ on $\gamma$ reflects that the influence of the heat released by the flame comes mainly through $\varepsilon$, which is proportional to $[\gamma-\tilde{L} /(1+s)]^{1 / 2}$ for given values of the gas and solid properties, rather than directly through $\gamma$. Sample streamlines, isotherms and the region of large enthalpy defect are displayed in figure 2 for $\gamma=5$ and $\varepsilon \approx 0.075(U=0.1)$. As can be seen, the flame extends to about seven radii ahead of the vaporization front in the particular case of figure 2 , and the radius of the flame soon becomes large compared with the radius of the rod and takes on the parabolic trend predicted by the asymptotic results of the previous section for $\varepsilon x \gg 1$. A large enthalpy defect $(H<0)$ exists in the vicinity of the rod upstream of the vaporization front, due to the heat loss toward the cold solid surface. Downstream of the vaporization front, for $x>0$, vaporization reduces the heat flux toward the solid and the enthalpy defect. The vaporization flux $m$, which is superimposed in figure 2, is maximum at the vaporization front and decreases very slowly for large values of $x$, in agreement with the asymptotic results. (These results would need modification if the fuel comprised only a thin coating of the rod). 


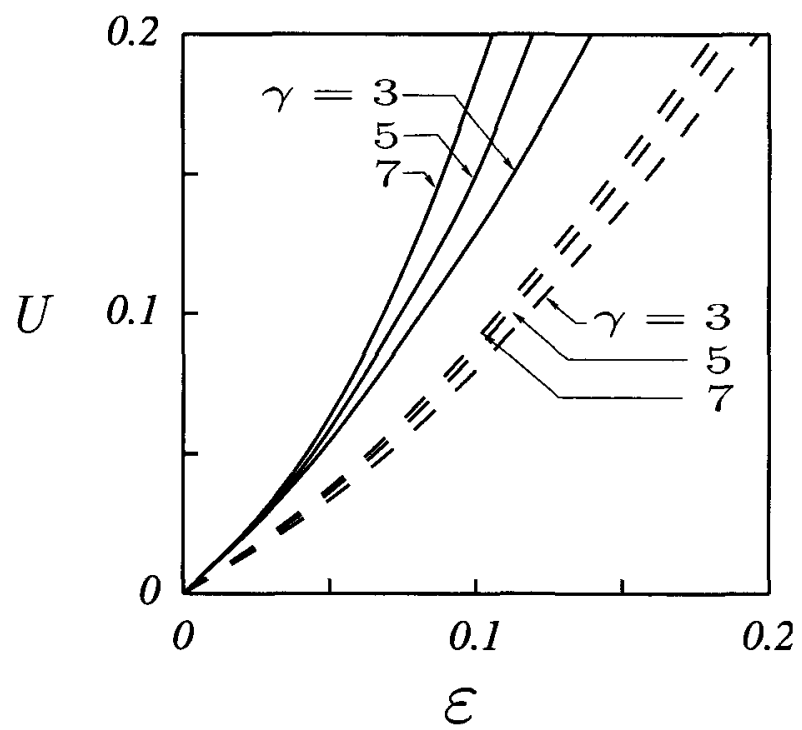

Figure 1. Nondimensional spread rate as a function of $\varepsilon$ for $s=8, \tilde{L}=2, \operatorname{Pr}=1$ and three different values of $\gamma$. The broken curves are results for a constant property gas.

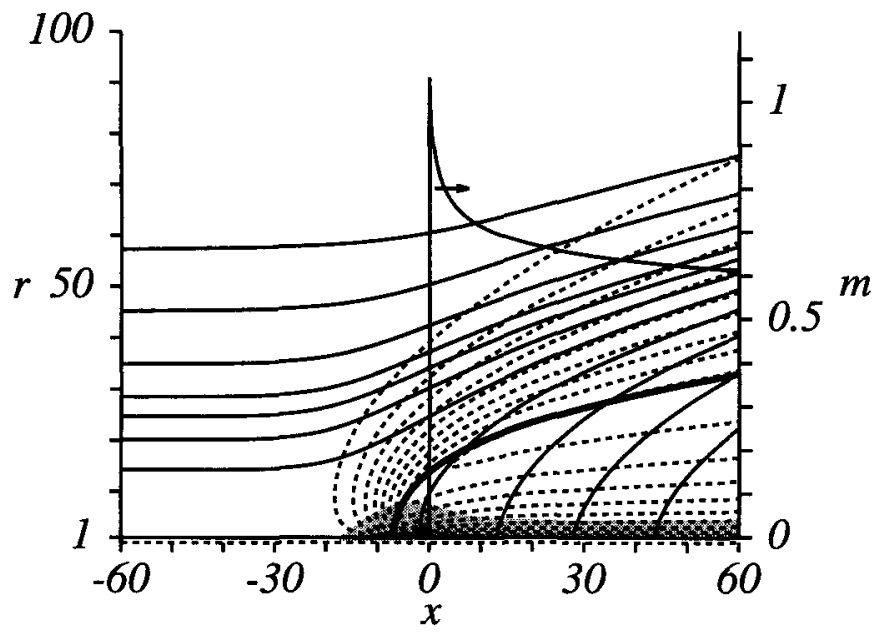

Figure 2. Full curves, streamlines -40 to 40 (step 10), 60, 100 and 160 (stream function defined by $\partial \psi / \partial r=r \rho u, \partial \psi / \partial x=-r \rho v$ and $\psi=0$ at $r=1, x<0$ ). Dotted curves, isotherms 1.5 to 5.5 , step 0.5 . Thick full curve, flame sheet. The shaded region corresponds to $H<-1$, the minimum value $H \approx-5$ being attained nearly at the foremost point of the flame on the solid surface. Values of vaporization flux $m(x)$ can be read on the right-hand scale.

The effect of the vaporization on the oncoming flow relative to the flame is therefore similar to that of a semi-infinite line of constant strength sources, inducing a velocity that slows down and deflects outwards the flow well upstream of the vaporization front. The thermal expansion of the gas around the flame adds to the effect of the vaporization, as can be seen by integrating the continuity equation across the thermal layer, from the rod $(r=1)$ outwards, in the region $\varepsilon x \gg 1$ where $u \approx U$, to obtain $\rho v r=m+\Delta m$ with $\Delta m=-U \int_{1}^{\infty}(\partial \rho / \partial x) r \mathrm{~d} r$. The 
parabolic growth of the radius of the flame, on which $T \rightarrow T_{f}$ for $\varepsilon x \rightarrow \infty$, amounts to a constant (up to logarithms, as for $m$ ) additional effective source strength $\Delta m$. The ratio $\Delta m / m$ increases with $\varepsilon$, from 0 to 4.8 in the range of figure 1 , and decreases slightly with increasing $\gamma$. In an attempt to assess the relative importance of the vaporization and the thermal expansion of the gas on the flame spread rate, problem (1)-(5) was solved for a constant property fluid $(\tilde{k}=\rho=1)$ using the same values of $s, \tilde{L}$ and $\operatorname{Pr}$ as before. The results are represented by the broken curves of figure 1 .

A gravitational acceleration $g$ acting in the direction of flame propagation would affect the gas flow in the transport region if $g$ were of the order of $g_{c}=\varepsilon^{3} \alpha_{g}^{2} / a^{3}$. When $g \gg g_{c}$ buoyancy induces a gas velocity of order $\left(g \alpha_{g}\right)^{1 / 3} \gg V_{p}$ in the transport region, whose size becomes $\alpha_{g}^{2 / 3} / g^{1 / 3}$, smaller than the estimate of $l_{g}$ given in the introduction. The energy balance $q_{g} l_{g} a \sim \rho_{s} c_{s} a^{2} V_{p} \Delta T_{s}$ leads to the modified estimate $V_{p}=\mathrm{O}\left[\left(\varepsilon \alpha_{g} / a\right)\left(g_{c} / g\right)^{1 / 3}\right]$ for $g>g_{c}$ but $\alpha_{g}^{2 / 3} / g^{1 / 3}$ still large compared with $a$; see [9]. The condition for the solid to behave as thermally thin in this range of $g$ is still $N \ll 1$. For comparison notice that, in the case of a slab, gravity affects the gas transport region when $g>g_{c 1}=\varepsilon^{6} \alpha_{g}^{2} / a^{3}$, but the order of magnitude of the spread rate remains $V_{p}=\mathrm{O}\left(\varepsilon^{2} \alpha_{g} / a\right)$ insofar as the solid is thermally thin $\left(a \ll\left(\alpha_{s} l_{g} / V_{p}\right)^{1 / 2}\right)$, which happens while $g<g_{c 2}=g_{c 1} /\left(\varepsilon^{6} N^{3}\right)$.

Longitudinal heat conduction in the solid extends to distances $x_{c}=\mathrm{O}\left(l_{g} \alpha_{s} / \alpha_{g}\right)$ upstream of the vaporization front, or larger than $x_{c}$ by a factor of $\left(g / g_{c}\right)^{2 / 3}$ if $g>g_{c}$. The formulation of the previous section relies on the assumption $x_{c} \ll l_{g}$, but there are cases of interest, when the rod has a metallic core, in which $x_{c}$ is of the order of or larger than $l_{g}$. Then longitudinal conduction in the solid affects the solution through its influence on the solid temperature appearing in condition $(4 a)$. In principle, the nondimensional energy equation $U \mathrm{~d} T_{s} / \mathrm{d} x=\left(\alpha_{s} / \alpha_{g}\right) \mathrm{d}^{2} T_{s} / \mathrm{d} x^{2}+2 \varepsilon^{2}\left(T_{v}-1\right) /\left(T_{f}-1\right) q_{s}$ should be used to compute the solid temperature for $x<0$, with $q_{s}=\tilde{k} \partial H /\left.\partial r\right|_{r=1}$, which is the form taken by (4b) for $x<0$. However, as can be seen by integrating this energy equation from $-\infty$ to $0^{+}$, the amount of heat that must reach the nonvaporizing surface in order to raise the temperature of the solid from the ambient temperature far upstream to the uniform vaporization temperature $T_{v}$ for $x \geqslant 0$ does not depend on the value of the solid conductivity. Moreover, the effect of the solid temperature distribution on the gas flow and the propagation speed is small for small values of $\left(T_{v}-1\right) /\left(T_{f}-1\right)$, and disappears under the approximation discussed in the last paragraph of the previous section. Thus the results obtained above, which do not depend on the value of the solid conductivity, should be applicable with good approximation to conducting rods. This conclusion holds for thermally thin rods only. The 'thermal thickness' of a rod made of an inert core and a combustible coating depends on the coating-to-core thickness and conductivity ratios, and experiments carried out by Bakhman et al [5] under normal gravity show that the spread rate is a function of these ratios.

\section{Conclusions}

The rate of flame spread over a thermally thin rod in the absence of gravity is higher by a factor of order $\left(\rho_{s} c_{s} \Delta T_{s} / \rho_{g} c_{p} \Delta T_{g}\right)^{1 / 2} \gg 1$ than the rate of flame spread over a slab in the same conditions, the latter being given by de Ris' formula in order of magnitude. This large factor is also the ratio of the characteristic radius of the advancing flame to the radius of the rod.

The ratios of surface-to-environment radiation to gas-to-surface conduction and gas-tosurface radiation to gas-to-surface conduction are reduced by a factor of the same order in comparison with their values for a slab.

At distances from the rod of the order of its radius, the flow relative to the steadily propagating flame is dominated by radial diffusion and radial convection due to the vaporization 
and the thermal expansion of the gas, while axial convection and diffusion come into play at distances of the order of the radius of the flame.

A gravitational acceleration in the direction of flame spread first influences propagation when the buoyancy-induced velocity around the flame front is of the order of the propagation speed. The propagation speed decreases as the inverse of the cubic root of the gravitational acceleration when it takes values above this threshold.

Axial conduction in the solid can extend to large distances upstream of the vaporization front in rods with a metallic core. However, the effect of axial conduction in the solid on the spread rate is typically small for thermally thin solids, because it only leads to temperature increments which are small compared with the temperature variations in the gas.

\section{Acknowledgments}

This work was supported by the Spanish DGICYT under grants PB95-0008 and PB94-0400, by INTA contract 4070-0036/1996 and by the Human Capital and Mobility Programme 'Gravity Dependent Phenomena in Combustion' of the CE.

\section{References}

Kosdon F J, Williams F A and Buman C 1969 Combustion of vertical cellulosic cylinders Proc. 12th Symp. on Combustion (Pittsburgh, PA: Combustion Institute) pp 253-64

Sibulkin M and Lee C K 1974 Flame propagation measurements and energy feedback analysis for burning cylinders Combust. Sci. Technol. 9 137-47

Fernández-Pello A C, Ray S R and Glassman I 1978 Downward flame spread in an opposed forced flow Combust. Sci. Technol. 19 19-30

Fernández-Pello A C and Santoro R J 1978 On the dominant mode of heat transfer in downward flame spread Proc. 17th Symp. on Combustion (Pittsburgh, PA: Combustion Institute) pp 1201-9

Bakhman N N, Aldabaev L I, Kondrikov B N and Filippov V A 1981 Burning of polymeric coatings on copper wires and glass threads: I. Flame propagation velocity Combust. Flame 41 17-34

Tewarson A and Khan M M 1988 Flame propagation for polymers in cylindrical configurations and vertical orientation Proc. 22th Symp. on Combustion (Pittsburgh, PA: Combustion Institute) pp 1231-40

Weber R O and de Mestre N J 1990 Flame spread measurements on single ponderosa pine needles: effect of sample orientation and concurrent external flow Combust. Sci. Technol. 70 17-32

Weber R O 1991 Dimensional analysis of the effect of gravitational acceleration on thermally controlled flame spread Combust. Sci. Technol. 79 157-60

Tarifa C S, Liñán A, Salvá J J, Juste G L, Tizón J M and Cura J M 1990 Study on combustion processes in reduced gravity Final ESA report LP TR 9004

de Ris J N 1969 Spread of a laminar diffusion flame Proc. 12th Symp. on Combustion (Pittsburgh, PA: Combustion Institute) pp 241-52

Bhattacharjee S and Altenkirch R A 1991 The effect of surface radiation on flame spread in a quiescent, microgravity environment Combust. Flame 84 160-9

Bhattacharjee S and Altenkirch R A 1990 Radiation-controlled, opposed-flow flame spread in a microgravity environment Proc. 23th Symp. on Combustion (Pittsburgh, PA: Combustion Institute) pp 1627-33

Olson S L, Ferkul P V and T'ien J S 1988 Near-limit flame spread over a thin solid fuel in microgravity Proc. 22th Symp. on Combustion (Pittsburgh, PA: Combustion Institute) pp 1213-22

West J, Tang L, Altenkirch R A, Bhattacharjee S, Sacksteder K and Delichatsios M A 1996 Quiescent flame spread over thick fuels in microgravity Proc. 26th Symp on Combustion (Pittsburgh, PA: Combustion Institute) pp 1335-43

Fernández-Pello A C and Williams F A 1977 A theory of flame spread over flat surfaces of solid combustibles Combust. Flame 28 251-77 